\title{
Flavor Oscillation of Neutrino with Mass Matrix
}

\author{
Jhulan Powrel \\ Department of Physics,Butwal Multiple Campus(TU),Butwal,Nepal \\ jhulan.sumi@gmail.com
}

\begin{abstract}
There is evidence that atmospheric, solar, accelerator, and reactor neutrinos changes from one flavor to another as observed in experiment.MiniBooNE and KamLAND are experimental set up used for their observations. Thus it is obvious that neutrino have mass hierarchy with changing flavor.
\end{abstract}

Keywords: neutrino mass matrix, neutrino flavor, mass hierarchy, standard model.

\section{INTRODUCTION}

The non baryonic dark matter includes neutrinos which were discovered as they have mass [4].The impact of matter on neutrino oscillations was first studied by Wolfenstein in 1978 [6]. He marked that matter suppresses oscillations of the solar neutrinos propagating in the Sun and supernova neutrinos inside a star. Similarly neutrino flavor evolution in the Earth is essentially oscillations in a multilayer medium with slowly changing density in the individual layers and sharp density change on the borders of layers.

\section{NEUTRINO FLAVOR OSCILLATION}

The phenomenon known as neutrino flavor oscillation, in which neutrinos are able to oscillate among the three available flavors while they propagate through space. Specifically, this occurs because the neutrino flavor eigenstates are not the same as the neutrino mass eigenstates. This allows for a neutrino that was produced as an electron neutrino at a given location to have a calculable probability to be detected as either a muon or tau neutrino after it has traveled to another location. There is evidence that atmospheric, solar, accelerator, and reactor neutrinos change from one flavor to another in accordance with the experiment in the KamLAND . However the MiniBooNE experiment concluded about the existence of sterile neutrino. Experimental data obvious that neutrino have masses which show hierarchy as they travel through matter or vacuum. If neutrinos have masses, then there is a spectrum of three or more neutrino mass eigenstates, $v_{1}, v_{2}$, $v_{3}, \ldots$, that are the analogues of the charged-lepton mass eigen states, e, $\mu$ and $\tau$.Thus, the neutrino state created in the decay $W^{+} \rightarrow \ell_{\alpha}^{+}+v$ is the state $\left|v_{\alpha}\right\rangle=\sum_{i} U_{\alpha i}^{*}\left|v_{i}\right\rangle$.

This superposition of neutrino mass eigen states [2], produced in association with the charged lepton of "flavor" $\alpha$, is the state that refer to as the neutrino of flavor $\alpha$.For any neutrino the mass eigen state $v_{i}$ as a superposition of the neutrinos of definite flavor given by $\left|v_{i}\right\rangle=\sum_{\beta} U_{\beta i}\left|v_{\beta}\right\rangle$. However there are only three known charged lepton mass eigen states[2] out of more then three neutrino mass eigen states. Thus, if there are four $v_{i}$, then one linear combination of them, $\left|v_{s}\right\rangle=\sum U_{\dot{s}}^{*}\left|v_{i}\right\rangle$ does not have a charged-lepton partner, and consequently does not couple to the Standard Model W boson. Such a neutrino, which does not have any Standard Model weak couplings, is referred to as a "sterile" neutrino. During the process of neutrino flavor change a neutrino born with flavor $\alpha$ becomes one of a different flavor $\beta$ while propagating in vacuum or in matter. This process, often referred to as neutrino oscillation, is quantum mechanical. In the probability $\left.P\left(v_{\alpha} \rightarrow v_{\beta}\right)=\mid A m p\left(v_{\alpha} \rightarrow v_{\beta}\right)\right)^{2}$ for the $\operatorname{oscillation}\left(v_{\alpha} \rightarrow v_{\beta}\right)$, only the relative phases of the propagation amplitudes, Prop $\left(v_{\mathrm{i}}\right)$ for different mass eigen states will have physical consequences. It is observed that the probability for oscillation of an antineutrino is the same as that for a neutrino [2], except that the mixing matrix $U$ is replaced by its complex conjugate given by,

$$
\begin{array}{r}
P\left(v_{\beta} \rightarrow v_{\alpha} ; U\right)=P\left(v_{\alpha} \rightarrow v_{\beta} ; U^{*}\right) \\
\text { and } P\left(\bar{v}_{\alpha} \rightarrow \bar{v}_{\beta} ; U\right)=P\left(v_{\alpha} \rightarrow v_{\beta} ; U^{*}\right) .
\end{array}
$$


When neutrinos travel through matter (e.g., in the Sun, Earth, or a supernova), their coherent forwardscattering from particles they encounter along the way can significantly modify their propagation [10]. As a result, the probability for changing flavor can be rather different than it is in vacuum [3]. Flavor change that occurs in matter, and that grows out of the interplay between flavor- non changing neutrinomatter interactions and neutrino mass and mixing, is known as the Mikheyev-Smirnov-Wolfenstein (MSW) effect. The effect of matter is illustrated by the propagation of solar neutrinos through solar matter. When combined with information on atmospheric neutrino oscillation, the experimental bounds on short-distance $(\mathrm{L} \leq 1 \mathrm{~km})$ oscillation of reactor $\bar{v}_{e}[1]$ shows that, if there are no sterile neutrinos, then only two neutrino mass eigenstates, $v_{1}$ and $v_{2}$, are significantly involved in the evolution of the solar neutrinos. Correspondingly, only two flavors are involved: the $v_{\mathrm{e}}$ flavor with which every solar neutrino is born and the effective flavor $v_{x}$ some linear combination of $v_{\mu}$ and $v_{\tau}$ which it may become (fig. 1).

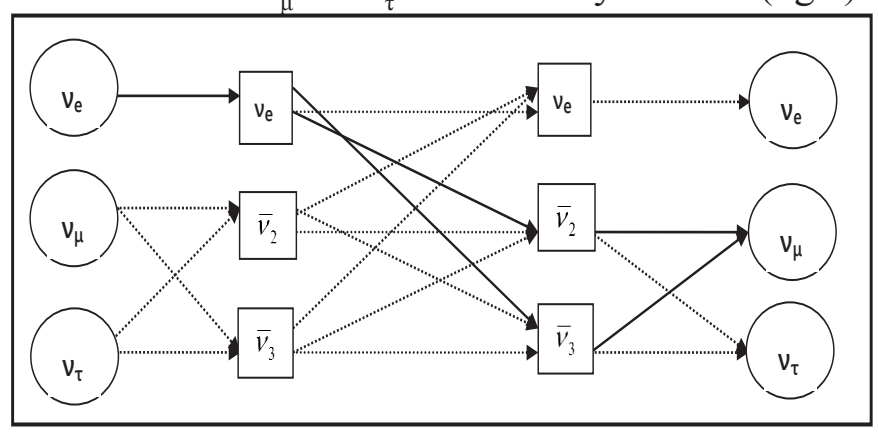

Fig 1: Scheme of transitions between the flavor states [6].

Evolution is considered in the propagation basis $\bar{v}$ .The lines which connect the flavor states and the propagation-basis states indicated projection of one basis onto another. The lines connecting the states of propagation basis $\bar{v}$ show transitions between them [6].

Historically, neutrino (or antineutrino) quasi-elastic scattering refers to the processes,

$v_{\mu} n \rightarrow \mu^{-} p$ and $\bar{v}_{\mu} p \rightarrow \mu^{+} n$,

where a charged lepton and single nucleon are ejected in the elastic interaction of a neutrino (or antineutrino) with a nucleon in the target material. This is the final state one would strictly observe, for example, in scattering off of a free nucleon target. QE scattering is important as it is the dominant neutrino interaction at energies less than about 1 $\mathrm{GeV}$ and is a large signal sample in many neutrino oscillation experiments.

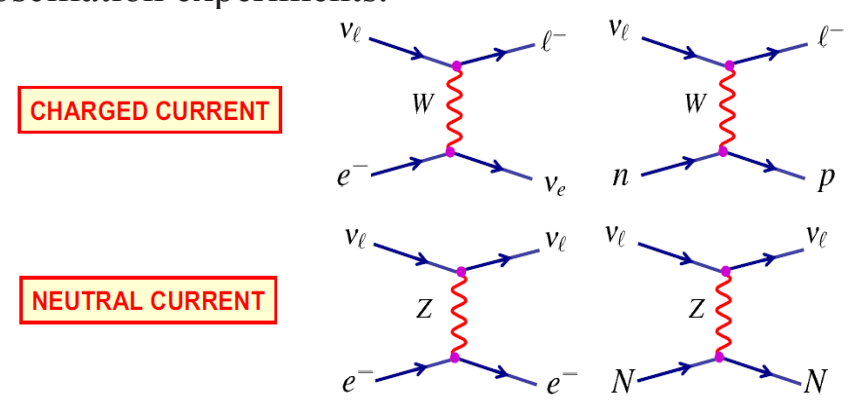

Fig 2: Interaction model [8]

The neutrinos interact with matter in two ways [8]; charged current (CC) interactions via a W-boson and neutral current (NC) interactions via a Z-boson against the target either atomic electrons or nucleons within the nucleus as in fig.2. Due to interaction [8] the neutrinos from the sun and nuclear reactions showing energy $E_{v} \approx 1 \mathrm{MeV}$ and those from atmospheric neutrinos have. $E_{v} \approx 1 \mathrm{GeV}$. Change of neutrino flavor, whether in matter or vacuum, does not change the total neutrino flux. Thus, unless some of the solar $v_{\mathrm{e}}$ are changing into sterile neutrinos, the total active high-energy flux measured by the neutral-current reaction $v+d \rightarrow v+p+n$, should agree with the predicted total solar neutrino flux based on calculations of neutrino production in the Sun. This agreement provides evidence that neutrino production in the Sun is correctly understood, further strengthens the evidence that neutrinos really do change flavor [5], and strengthens the evidence that the previously-reported deficits of solar $v_{\mathrm{e}}$ flux (solar neutrino problem) are due to this change of flavor. Predictions for proton decay are changed within SUSY GUTs. The increased unification scale with respect to the minimal $\mathrm{SU}(5)$ results in a bigger $M_{x}$ mass. This results in a substantially increased lifetime for the proton of about $10^{35}$ years, which is compatible with experiment. The experimentally determined lower limit of the proton lifetime of $\tau_{p}>1.9 \times 10^{3}$ years for this channel is less restrictive than the $p \rightarrow \pi^{0}+e^{+}$mode. 


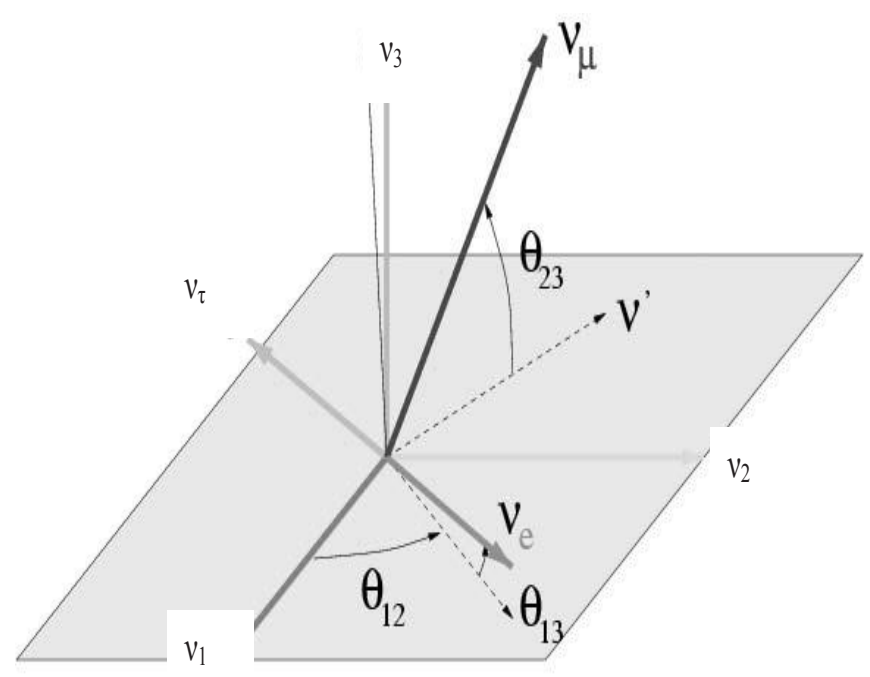

Fig.3.Graphical representation of the mixing matrix elements between flavor and mass eigen states [11].

Recent calculations[11] within SUSY SU(5) and SUSY SO(10) seems to indicate that the upper bound on the theoretical expectation is $\tau_{p}>1.9 \times 10^{3}$ years which should be well within the reach of longer running Super-K and next-generation experiments like Hyper-Kamiokande, ICARUS etc. Other dominant decay modes might reveal in some left-right symmetric models, which prefer $p \rightarrow \mu^{+} K^{0}$.

The experimental bound here is $\tau_{p}>1.9 \times 10^{3}$ years, for a bound on Rp-violating constants coming from proton decay. After discussing the standard model and its possible extensions let us take a look at what type of neutrino mass generation can be realized. In accordance with Standard Model there is no mass for neutrino because of lepton and baryon number conservation, but experimental evidence supported that a neutrino posses Majorana mass term which indicates the physics 'beyond the standard model [11] i.e. neutrino masses can be generated within the gauge structure of $\mathrm{SU}(3) \otimes$ $\mathrm{SU}(2) \times \mathrm{U}(1)$ by enlarging the particle content or adding non-renormalizable interactions. Even by adding new particles this sometimes is nevertheless still called 'standard model' because the gauge structure is unchanged. Neutrino masses can be created in the standard model by extending the particle content of the theory. Dirac mass terms and the corresponding Yukawa couplings can be written for neutrinos if singlet $v_{R}$ are included in the theory as for all other fermions. This would result in

$$
\mathcal{L}_{\mathrm{Yuk}}=-c_{\nu} \bar{v}_{R} \phi^{\dagger}\left(\begin{array}{c}
\nu_{e_{L}} \\
e_{L}
\end{array}\right)+\text { h.c. }=-c_{\nu} v \bar{v} \nu \text {. }
$$

The smallness of the neutrino mass must then be explained by a correspondingly smaller Yukawa

coupling $c_{v}$. It is obvious that in the minimal SU (5) neutrinos remain mass less. Again the observation of oscillations between different flavors of neutrinos from the Sun, nuclear reactors and cosmic rays shows that the two differences in the square of the masses of the three types of neutrinos of definite mass are $8.0_{-0.3}^{+0.4} \times \mathbb{0 0} 0^{-5} \mathrm{eV}^{2}$ and between 1.9 and 3.0 times $10^{-3} \mathrm{eV}^{2}$. Thus the neutrino masses are all much less than $1 \mathrm{eV}$ unless they are highly degenerates[9]. The observation for $\mathrm{CMB}$ anisotropies and large scale structure shows that the sum of the three neutrino masses is less than $0.68 \mathrm{eV}$, so if they are degenerate the common mass is less then $0.23 \mathrm{eV}$ which is in contrary to the value $1.2 \mathrm{eV}$ for the sum of the neutrino masses obtained from neutrino less double beta decay experiment.

But, as in the standard model, enlarging the Higgs sector allows us to introduce Majorana mass terms. An attempt is made to generate the bimaximal mixings (as in fig.3) of the three species of neutrinos from the textures of the right-handed Majorana neutrino mass matrices. Patrgiri,M. et al(2003) suggested a paper for the generation of the nearly degenerate as well as the inverted hierarchical models of the left-handed Majorana neutrino mass matrices using the non-diagonal textures of the right-handed Majorana neutrino mass matrices and the diagonal form of Dirac neutrino mass matrices, within the framework of the see-saw mechanism in a model independent way[7]. Such Majorana neutrino mass models are important in explaining the recently reported result on the neutrino less double beta decay (ov $\beta \beta$ ) experiment, together with the earlier established data on LMA MSW solar and atmospheric neutrino oscillations. In recent day, many group attempt to generate the degenerate 
as well as the inverted hierarchical pattern of the left-handed Majorana neutrino mass matrices using the see-saw mechanism in a model independent way. A group of scientist, Patgiri M.et al [7] works with neutrino mixings which are provided from the texture of the right-handed Majorana mass matrix $\mathrm{M}_{\mathrm{RR}}$, while keeping the Dirac neutrino mass matrix $\mathrm{m}_{\mathrm{LR}}$ in the diagonal form. They have taken the Dirac neutrino mass matrix $\mathrm{m}_{\mathrm{LR}}$ as either the charged lepton mass matrix $\mathrm{m}_{\mathrm{LR}}=\tan \beta \mathrm{m}_{l}$ or the up-quark mass matrix $\mathrm{m}_{\mathrm{LR}}=\mathrm{m}_{\text {up }}$. The left-handed Majorana neutrino mass matrix $\mathrm{m}_{\mathrm{LL}}$ is given by the celebrated see-saw formula $m_{\mathbb{L}}=-m_{R} M_{R}^{-1} m_{R}^{T}$, where $\mathrm{m}_{\mathrm{LR}}$ is the Dirac neutrino mass matrix in the leftright (LR) convention. The leptonic (MNS) mixing matrix is now given by

$$
\begin{aligned}
& V_{\mathrm{MNS}}=V_{\nu \mathrm{L}}^{\dagger} \\
& \text { where } m_{L}^{\text {diag }}=V_{v L} m_{L} V_{v L}^{T} .
\end{aligned}
$$

Here both $\mathrm{m}_{\mathrm{LR}}$ and the charged lepton mass matrix $\mathrm{m}_{1}$ are taken as diagonal, whereas the right-handed Majorana neutrino mass matrix $\mathrm{M}_{\mathrm{RR}}$ is non-diagonal. Using the see-saw formula it generates both patterns of $\mathrm{m}_{\mathrm{LL}}$ nearly degenerate and inverted hierarchical neutrino mass models.

\section{EXPERIMENTAL LIMITS}

The neutrino mass cannot be discussed in isolation since, it is related to other issues of which the most important is that of neutrino mass mixing.In order to get some limits on the masses one can use kinematical considerations on different types of reactions, $n \rightarrow p+e+\bar{v}_{e}$

so, it is possible to establish an upper bound for the $v_{\mathrm{e}}$ mass. Upper bounds for the $v_{\mu}$ and $v_{\tau}$ are treated as $\pi^{+} \rightarrow \mu^{+}+v_{\mu}$ and $\tau \rightarrow 2 \pi^{+}+3 \pi^{-}+\pi^{0}+v^{\tau}$ . The experimental finding on the upper limits on neutrino masses

$$
\begin{aligned}
& \mathrm{m}_{v_{e}}<3 \mathrm{eV}, \\
& \mathrm{m}_{v_{\mu}}<190 \mathrm{KeV}, \\
& \mathrm{m}_{v_{\tau}}<18.2 \mathrm{MeV} .
\end{aligned}
$$

Only the mass eigen states have well defined masses and not the flavor eigen states. The above values are valid only when there is no mixing at all, i.e.the flavor eigen states are equal to the mass eigen states.

\section{CONCLUSION}

Several experimental evidences as per [1],[5],[6] and[7] and the data obtained from KamLAND and MiniBooNE experiment confirm the existence of sterile neutrino(referred as beyond standard model) and Majorana neutrino with changing flavor. The study also observes the fitness of sea saw mechanism for the explanation of neutrino mass matrix.

\section{REFERENCES}

[1] Achkar,B.et al. (1995).Nuclear physics. The Bugey Collaboration, B434, 503.

[2] Amsler,C. et al.(2008).Physics letters B667. Fermilab:USA. [URL:http://pdg.lbl.gov/]

[3] Apollonio,M. et al.(2002). Oscillation physics with a neutrino factory. CERN, Switzerland.

[CERN, 2002-208, hep-ph/0210192]

[4] Aryal,B.(2010) Galaxy and Cluster Evolution (Lec.4),Summer school A\& A,Kathmandu.

[5] Beringer, J. et al. (2012). Rudolf peierls centre for theoretical physics. F e r m i $1 \mathrm{ab}$ : University of Oxford.

[6] Blennow, M.et al. (2013). Advances in High Energy Physics: Neutrino propagation in matter. (Rev. Art .Vol. 2013, ID 972485) Hindawi Publishing Corporation: Germany. [http://dx.doi.org/10.1155/2013/972485]

[7] Patgiri,M.et al. (2003), International Journal of Modern Physics: Right-handed major a n a neutrino mass matrices forgenerating bimaximal mixings in degenerate and inverted models of neutrinos. (Vol.18, No.5,743/753)World Scientific Publishing Company: New Jersey and London.

[8] Thomson, M.A. (2009), Michaelmas Term 2009(Paper), Particle physics, Particle physics group. [http://pdg.lbl.gov]

[9] Weinberg,S.(2008),Cosmology, Thermal history, Oxford University press: New York.

[10] Wolfenstein, L.(1978).Physical Review (Particles and Fields),Neutrino oscillations in matter. [http://adsabs.harvard.edu/abs/1978PhRvD.17.2369W]

[11] Zuber,K.(2004), Neutrino physics, Taylor \& Francis Group: New York. 